\title{
LRP5 variants may contribute to ADPKD
}

\author{
Wybrich R Cnossen ${ }^{1}$, René HM te Morsche ${ }^{1}$, Alexander Hoischen ${ }^{2}$, Christian Gilissen ${ }^{2}$, Hanka Venselaar ${ }^{3}$, \\ Soufi Mehdi ${ }^{4}$, Carsten Bergmann ${ }^{5,6}$, Monique Losekoot ${ }^{7}$, Martijn H Breuning ${ }^{7}$, Dorien JM Peters ${ }^{8}$, \\ Joris A Veltman ${ }^{2,9}$ and Joost PH Drenth ${ }^{\star, 1}$
}

\begin{abstract}
Mutations in Polycystic Kidney Disease proteins (PKD1 or PKD2) are causative for autosomal dominant polycystic kidney disease (ADPKD). However, a small subset of ADPKD probands do not harbor a mutation in any of the known genes. Low density lipoprotein Receptor-related Protein 5 (LRP5) was recently associated with hepatic cystogenesis in isolated polycystic liver disease (PCLD). Here, we demonstrate that this gene may also have a role in unlinked and sporadic ADPKD patients. In a cohort of 79 unrelated patients with adult-onset ADPKD, we identified a total of four different LRP5 variants that were predicted to be pathogenic by in silico tools. One ADPKD patient has a positive family history for ADPKD and variant $L R P 5$ c.1680G $>$; p.(Trp560Cys) segregated with the disease. Although also two PKD1 variants probably affecting protein function were identified, luciferase activity assays presented for three LRP5 variants significant decreased signal activation of canonical Wnt signaling. This study contributes to the genetic spectrum of ADPKD. Introduction of the canonical Wnt signaling pathway provides new avenues for the study of the pathophysiology.
\end{abstract}

European Journal of Human Genetics (2016) 24, 237-242; doi:10.1038/ejhg.2015.86; published online 29 April 2015

\section{INTRODUCTION}

Autosomal dominant polycystic kidney disease (ADPKD; MIM $173900)$ is the most common renal Mendelian disorder with a global prevalence of $1 / 400-1 / 1000 .^{1}$ Development of multiple fluid-filled cysts may cause bilateral enlarged kidneys and result in end-stage renal disease. Renal replacement therapy is necessary in about $\sim 5 \%$ of ADPKD patients. ${ }^{1}$ In addition, the majority of ADPKD patients have health-care issues owing to extrarenal features and complications such as polycystic liver disease (PCLD; 83\%), hypertension (80\%), cardiac valve abnormalities (25\%), intracranial aneurysm (10\%) and cysts in the pancreas and other organs. ${ }^{2,3}$

There is a broad clinical expression of (extra-)renal manifestations and symptoms among ADPKD patients. ${ }^{1,3}$ The phenotype of affected individuals from single large pedigrees may cover the complete spectrum of ADPKD ranging from few renal cysts to grossly enlarged polycystic kidneys with renal failure. ${ }^{4}$ These observations suggest that modifying factors, in trans or multiple PKD mutations may influence the onset and course of renal cystogenesis.,

The genotype-phenotype correlation is not completely understood in ADPKD. ${ }^{7}$ Linkage studies identified two disease-causing genes, Polycystic Kidney Disease 1 protein (PKD1; Chr.16p13.3; NG_ 008617.1) and Polycystic Kidney Disease 2 protein (PKD2; Chr.4q22.1; NG_008604.1). ${ }^{8,9}$ Molecular screening may confirm the clinical diagnosis with a mutation frequency of $76 \%$ PKD1 and $13 \%$ PKD2. ${ }^{10,11}$ Despite thorough screening, $\sim 11 \%$ of families remain unlinked to either locus, which suggest genetic heterogeneity. ${ }^{10,11}$
Approximately $10 \%$ of ADPKD patients have no family history of renal disease. ${ }^{12}$ These solitary cases may have mild or severe polycystic kidneys owing to incomplete disease penetrance, de novo (modifier) gene variants and environmental influences.

Recently, we identified the Low density lipoprotein Receptor-related Protein 5 (LRP5) gene (Chr.11q13.2; NG_015835.1) as the third locus associated with isolated PCLD (PCLD; MIM 174050) by whole-exome sequencing in an extended family. ${ }^{13}$ As PCLD is the most common extra-renal feature in ADPKD patients, we hypothesized that LRP5 variants may contribute to hepatic and renal disease heterogeneity in ADPKD. We addressed the role of LRP5 by screening all 23 exons in 79 unlinked and/or sporadic ADPKD patients, and performed some functional analyses.

\section{MATERIALS AND METHODS}

Patients

This study was approved by the institutional review board and ethics committee of the Radboud university medical center, Nijmegen. All participants provided informed consent. The families were recruited in The Netherlands, screened according to the Ravine criteria ${ }^{14}$ and blood samples were collected.

Polycystic kidney disease mutation analysis

Mutation screening of PKD1 (NM_001009944.2) and PKD2 (NM_000297.2) involved Sanger sequencing of exons and flanking intronic regions and multiplex ligation-dependent probe amplification analysis in all probands by using bidirectional Sanger sequencing on ABI3730 Genetic Analyzers (Applied Biosystems, Waltham, MA, USA). PKD1 variants were assessed using the

\footnotetext{
${ }^{1}$ Department of Gastroenterology and Hepatology, Institute for Genetic \& Metabolic Disease (IGMD), Radboud Institute for Molecular LifeSciences (RIMLS), Radboud university medical center, Nijmegen, The Netherlands; ${ }^{2}$ Department of Human Genetics, Institute for Genetic \& Metabolic Disease (IGMD), Radboud Institute for Molecular LifeSciences (RIMLS), Radboud university medical center, Nijmegen, The Netherlands; ${ }^{3}$ Center for Molecular and Biomolecular Informatics, Institute for Genetic \& Metabolic Disease (IGMD), Radboud Institute for Molecular LifeSciences (RIMLS), Radboud university medical center, Nijmegen, The Netherlands; ${ }^{4}$ Department of Gastrointestinal and Oncological Surgery, Faculty of Medicine, University Mohammed First, Oujda, Morocco; ${ }^{5}$ Center for Human Genetics, Bioscientia, Ingelheim, Germany; ${ }^{6}$ Department of Nephrology and Center for Clinical Research, University Hospital Freiburg, Freiburg, Germany; ${ }^{7}$ Department of Clinical Genetics, Leiden University Medical Center, Leiden, The Netherlands; ${ }^{8}$ Department of Human Genetics, Leiden University Medical Center, Leiden, The Netherlands; ${ }^{9}$ Department of Clinical Genetics, Maastricht University Medical Centre, Maastricht, The Netherlands

${ }^{*}$ Correspondence: Dr JPH Drenth, Department of Gastroenterology and Hepatology, Radboud university medical center, P.O. Box 9101 , Nijmegen 6500 HB, The Netherlands. Tel: +31 24 3614760; Fax: +31 24 3540103; E-mail: joostphdrenth@cs.com

Received 20 September 2014; revised 27 February 2015; accepted 27 March 2015; published online 29 April 2015
} 
Autosomal Dominant Polycystic Kidney Disease mutation database v.3.0, http://pkdb.mayo.edu/. ${ }^{15}$ Protein Kinase C Substrate $80 \mathrm{~K}-\mathrm{H}$ (PRKCSH; NM_002743.3) and SEC63 homolog (SEC63; NM_007214.4) were analyzed in two probands with severe polycystic livers (109 from family A and 101 from family D) and patient 102 (family B) was screened for Polycystic Kidney and Hepatic Disease 1 protein (PKHD1; NM_138694.3) and Hepatocyte Nuclear Factor-1-beta (HNF-1 $\beta$; NM_000458.2).

\section{LRP5 sequencing and in silico analysis}

All 23 exons of the LRP5 gene (NM_002335.3) were screened using High Resolution Melting followed by Sanger sequencing. Scoring of LRP5 variants was performed with homology modeling, in silico analysis such as PolyPhen2, Mutpred, SIFT, Align GVGD, PhyloP and the Grantham score. Genome-wide sequence data from the 1000 Genomes Project, 6500 individuals from the National Heart, Lung and Blood Institute Exome Sequencing Project (version 0.0.27), 500 individuals from the Genome of The Netherlands, exome data from 2000 individuals of predominantly European ancestry sequenced inhouse, and DNA samples from 525 Moroccan healthy, unrelated individuals served as controls.

We reported one LRP5 variant (rs724159825) and three PKD1 variants (rs724159824; rs724159822; rs724159823) described in this manuscript to the public dbSNP database.

\section{Immunofluorescence and qPCR}

Subsequently, immunofluorescence studies, luciferase activity assays and qPCR experiments with wild-type (WT) and four mutant LRP5 constructs were conducted. Transfected HeLa cells were stained in order to elucidate the colocalization of LRP5 to the endoplasmic reticulum, Golgi apparatus and the plasma membrane (see Supplementary Information).

\section{Luciferase activity assay and real-time-PCR}

Wnt signaling activity was assessed using the Cignal Reporter TCF/LEF Assay Kit (Qiagen, Hilden, Germany) after transfecting CHO or HEK293 cells with WT or mutant constructs and addition of hWnt3a (R\&D Systems, Abingdon, UK).

Expression levels of Wnt target genes were assessed in Wnt3a-activated and non-activated transfected HEK293 cells by real-time-PCR.

\section{RESULTS}

A subset of 29 ADPKD patients had genotypic prescreening. Molecular diagnostics was performed for PKD1, PKD2 and PKHD1 (Chr.6p12.2; NG_008753.1) in adults. Additional analysis of HNF1ß (Chr.17q12; NG_013019.1) was performed in adolescents when genotype screening of $P K D 1, P K D 2$ and $P K H D 1$ were negative. No point mutations, $\mathrm{CNV}$ or large deletions were identified and all 29 affected individuals were designated as being unlinked. A group of 50 patients had a clinical diagnosis of ADPKD with a symptomatic polycystic liver. Variants for PCLD in PRKCSH (Chr.19p13.2; NG_009300.1) and SEC63 (Chr.6q21; NG_008270.1) affecting protein function were excluded, but the PKD genotype was unknown in this group. In this heterogeneous cohort of 79 ADPKD patients, we identified four LRP5 variants by Sanger sequencing (Table 1). Both unique LRP5 variants were detected in the subgroup of ADPKD with a severe polycystic liver. Here, we present the data of three adult-onset ADPKD patients and one adolescent ADPKD patient (Figure 1 and Supplementary Figures S1-S3).

\section{Family A}

The proband (109) presented to us with a symptomatic polycystic liver and renal cysts of various sizes at 51 years of age (Table 1). She was clinically diagnosed as ADPKD. Mutational screening of PRKCSH and SEC63 yielded no variants causing hepatic and renal cystogenesis. She was from Moroccan ancestry and her ancestors did not have renal disease suggestive for ADPKD. During the first pregnancy of her oldest daughter (202), polycystic kidneys and several small hepatic cysts were detected by abdominal ultrasonography. Renal failure was absent and mild hypertension was under control by use of an AT2-receptor antagonist. Both patients fulfilled the unified Ravine criteria for ADPKD. ${ }^{14}$

By LRP5 variant analysis, we identified a unique missense variant c.1680G > T; (p.(Trp560Cys); rs377144001) in both Moroccan patients (109 and 202). This LRP5 variant is not detected in genome-wide sequence data such as 1000 Genomes Project, and 2000 in-house exome sequencing data from patients of European ancestry. In addition, LRP5 c.1680G $>T$ is neither detected in 4400 AfricanAmerican exome sequencing cohort from the National Heart, Lung and Blood Institute (NHLBI) Exome Sequencing Project (ESP). This variant was recently identified once in an ESP European-American cohort with a total allele frequency of 1/12 988 (rs377144001, dbSNP v.138). The variant $L R P 5$ c. $1680 G>T$ was not present among 525 tested Moroccan DNAs of unrelated individuals.

In silico analysis predicts this variant to be pathogenic and alters a conserved LRP5 amino acid (Table 2). Homology modeling indicated that this missense variant affects one of the tryptophans that are important for the core structure of this domain. Multiple hydrophobic interactions and a hydrogen bond will be lost by the tryptophane amino acid change into cysteine. This variant is highly likely to change the secondary and tertiary structure of the protein, and to disturb the function of the second WD40 domain function.

Variants in the genes PKD2, PKHD1, PRKCSH and SEC63 affecting the protein function were absent, but molecular diagnostics of PKD1 revealed two unique variants. Both PKD1 variants, c.1281_ 1283delGGC; p.(Ala428del) (rs724159824) in exon 6 and c.3133G >C; p.(Val1045Leu) (rs724159822) in exon 13, were present in the proband (109). The p.(Ala428del) had been transmitted to the 33-year-old affected daughter (202), while the 27-year-old healthy daughter (203) inherited p.(Val1045Leu). In addition, the 25-year-old

Table 1 Patient characteristics

Baseline characteristics

At first presentation (diagnosis)

\begin{tabular}{|c|c|c|c|c|c|c|c|}
\hline Family & Subject & Age (years) & Sex & Age (years) & Creatinin $(\mu \mathrm{mol} / \mathrm{l})$ & $E S R D$ & $\begin{array}{l}\text { Treatment for polycystic kidneys } \\
\text { Medical/ Surgical }\end{array}$ \\
\hline \multirow[t]{2}{*}{ A } & 109 & 56 & $\mathrm{~F}$ & 51 & 155 & Yes & Thiazide and AT2-receptor antagonist; 9-months octreotide \\
\hline & 202 & 33 & $\mathrm{~F}$ & 24 & 79 & No & Antihypertensive treatment \\
\hline B & 101 & 60 & M & 55 & 80 & No & Antihypertensive treatment \\
\hline C & 102 & 18 & $\mathrm{~F}$ & 14 & 64 & No & - \\
\hline D & 101 & 51 & $\mathrm{~F}$ & 43 & 114 & Yes & Antihypertensive treatment; kidney transplantation \\
\hline
\end{tabular}


a

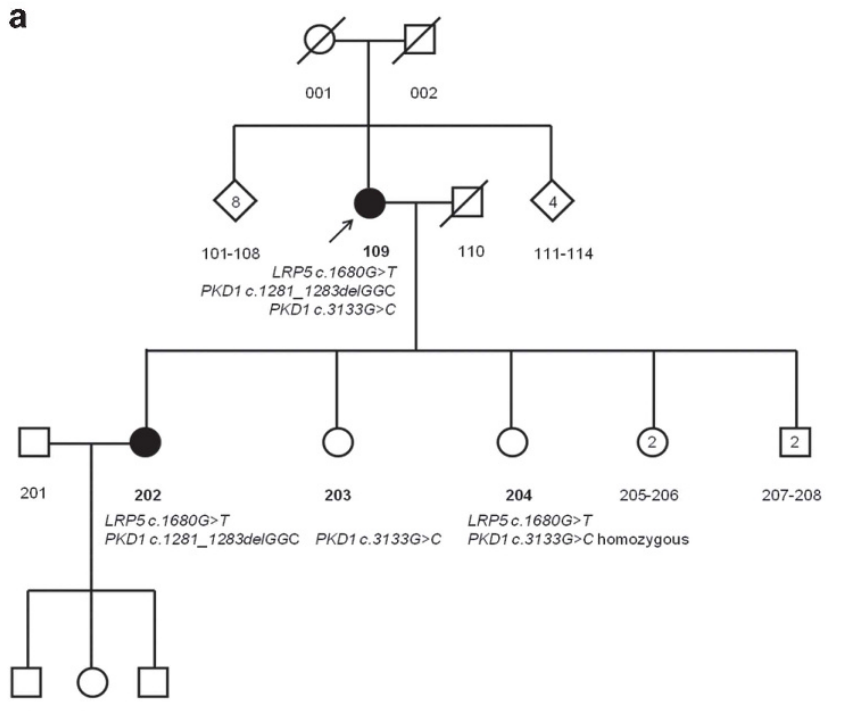

C

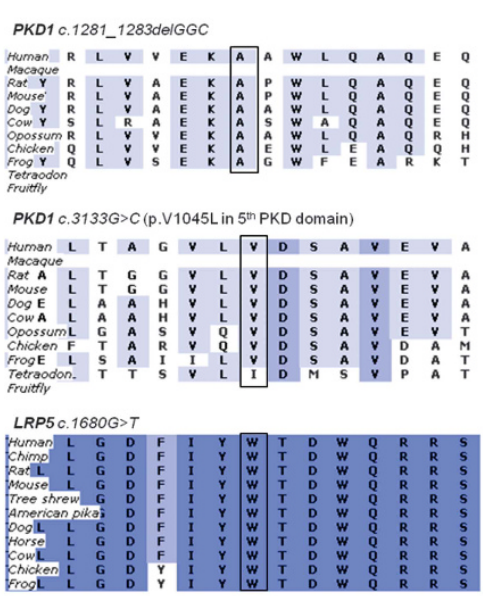

d

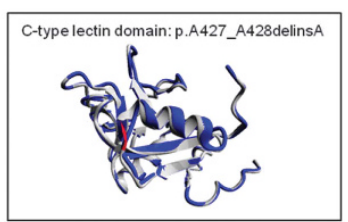

(no model available) e

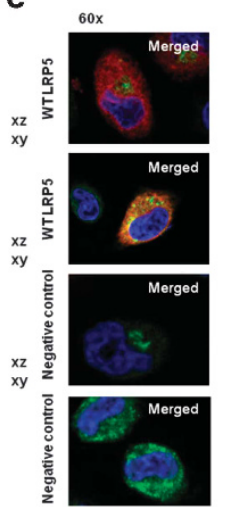

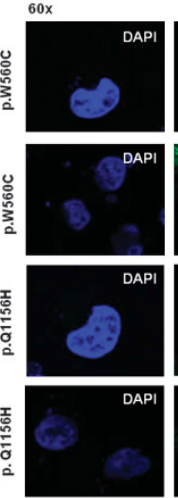
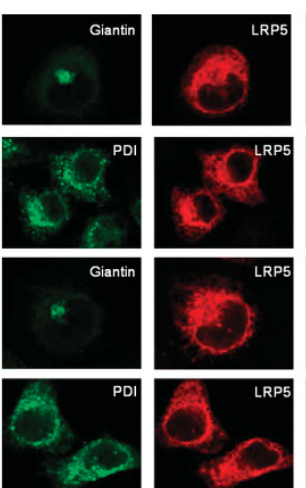
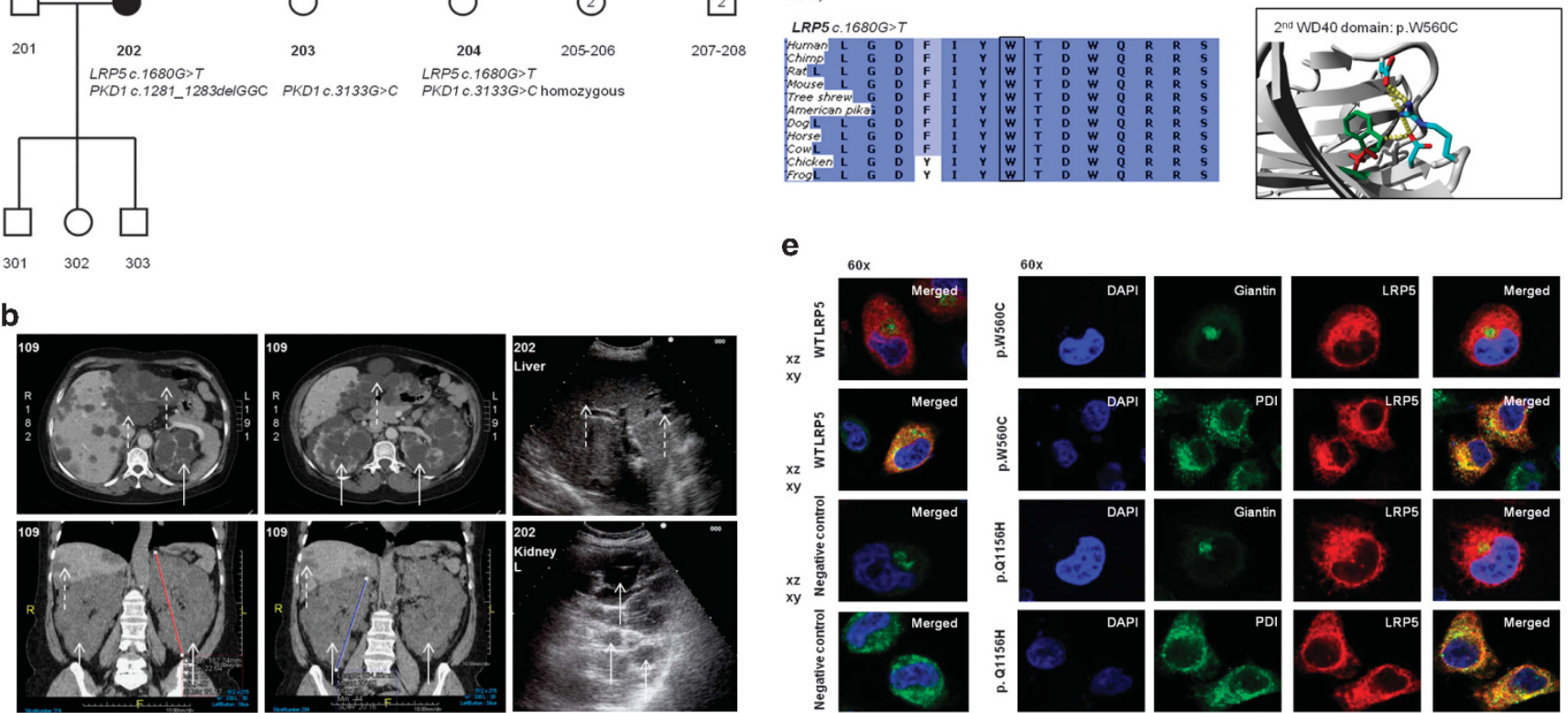

Figure 1 Clinical and genetic data of ADPKD family A. (a) Pedigree of family A present two affected individuals, proband 109 and daughter 202. Affected individuals indicate those with confirmed ADPKD on CT scanning or abdominal ultrasound according to the Ravine criteria. (b) CT scanning in proband 109 presented a severe polycystic liver in segments 2,3 and 8 and the kidney sizes were craniocaudal $19 \mathrm{~cm}$ (left) and $21 \mathrm{~cm}$ (right) in diameter. Abdominal ultrasonography in patient 202 assessed polycystic kidneys (white arrows) and multiple small hepatic cysts (dotted white arrows). (c) PKD1 c.1281_1283de/GGC and PKD1 c.3133G>C were detected in the proband. Both PKD1 variants are located in low evolutionary conserved regions in contrast to LRP5 c.1680C > T. (d) The in-frame PKD1 deletion is located on an extracellular C-type lectin domain at the end of a loop close to a double helix structure. The alanine deletion shortens the strand, but there is no destabilization of the domain which is closely located to a rigid double helix structure with many hydrogen bounds. Missense variant PKD1 p.(Val1045Leu) is located on the fifth tandem PKD domain without a difference in charge between WT and mutant protein. (e) Confocal imaging studies in transiently transfected HeLa cells identified co-localization of LRP5 in the endoplasmic reticulum. There were no differences between LRP5 localization compared with the WT LRP5 construct for all 4 LRP5 variants.

healthy daughter (204) harbors also p.(Val1045Leu), but in a homozygous state (PKD1 c.3133G>C homozygous). Both PKD1 variants are unreported in the literature (unclassified variants), online and inhouse databases and are localized in evolutionarily moderately low conserved domains.

The PKD1 missense variant p.(Val1045Leu) may be a benign variant, as this variant had been transmitted to the unaffected daughter (203). The consequence of this PKD1 variant is currently unclear, but non-penetrance or late-onset of ADPKD may play a role. The contributing effect of p.(Ala428del) is unclear, but homology modeling suggests that the nascent residues surrounding codon 248 are not conserved and that the C-type lectin domain is not pathogenic altered. It is well conceivable that the identified changes in this patient may exhibit their full pathogenic effect in concert.

Family B

A 57-year-old Dutch male came to our attention because of uncontrolled hypertension. Extensive clinical and radiological examination demonstrated large, complicated (multilocular) renal cysts (Supplementary Figure S1).

There was no family history for polycystic diseases, liver or renal disease. Therefore, no consent was retrieved for phenotype and genotype screening in family members. PKD1 and PKD2 variants were absent in the proband and Sanger sequencing of the LRP5 gene identified c.3107G $>A$; p. $(\operatorname{Arg} 1036 \mathrm{Gln} ; \mathrm{rs61889560)})$. This variant reported an incidence of $0.001-0.005(1 / 245)$ in public databases. The missense variant is located at the fourth WD40 and may lead to a less stabilized domain owing to loss of hydrogen and ion binding.

\section{Family C}

A 14-year-old female (102) was diagnosed with two large, symptomatic cysts with a diameter of $4.5 \mathrm{~cm}$ each and a small ('intrapolar') cyst was present in the left kidney with a normal right kidney (Supplementary Figure S2). Creatinine levels remained between 61 and $71 \mu \mathrm{mol} / \mathrm{l}$ within the normal range $(50-90 \mu \mathrm{mol} / \mathrm{l})$ during 4 years of follow-up. Her blood pressure was $130 / 70 \mathrm{~mm} \mathrm{Hg}$ and regularly 
monitored at home. Family history was negative for ADPKD, autosomal recessive polycystic kidney disease and PCLD or other genomic disorders, but her mother was diagnosed at the age of 30 with nephrotic syndrome of unknown cause. Both parents were screened and there were no structural renal abnormalities on ultrasonography.

Molecular diagnostics showed no evidence for the diagnosis of ADPKD or autosomal recessive polycystic kidney disease by analyses of the PKD1, PKD2, PKHD1 and HNF1 $\beta$ gene. We identified the LRP5 variant c.3403C > T; p.(Arg1135Cys; rs143396225). This variant is reported with a frequency of $1 / 1300$ in the European American ESP cohort from the NHLBI, and absent in the 1000 Genomes Project dataset and 2000 DNA exomes sequenced in-house. In silico analyses predicted LRP5 c.3403C $>T$ to be disease-associated with pathogenic effects for the protein function (Table 2). Homology modeling showed that the arginine is located at the fourth WD40 domain. The p. (Arg1135Cys) variant results in a change of a hydrophilic molecule to an apolar hydrophobic amino acid which may contribute to a destabilized WD40 domain. The loss of ionic interactions is likely to affect the function of the protein domain.

\section{Family D}

Bilateral enlarged kidneys containing multiple fluid-filled cysts were an accidental finding on ultrasonography in the proband (101) at the age of 43 (Supplementary Figure S3). Her blood pressure was under control $(128 / 70 \mathrm{~mm} \mathrm{Hg})$ with an antihypertensive ACE-inhibitor and she used antacid medication. Eight years after identification of polycystic kidneys, the 51-year-old patient received renal transplant from a living unrelated donor.

Family history for polycystic or renal diseases was unremarkable. No PRKCSH or SEC63 variants affecting the protein function were present, but $P K D$ screening revealed two $P K D 1$ variants; c.8293C $>T$; p.(R2765C) (rs144979397) on exon 23 and PKD1 c.11554delC; p.(L3852Wfs $\left.{ }^{\star 93}\right)$ (rs724159823) on exon 42. The mother also harbors the PKD1 missense variant $\mathrm{p} .(\mathrm{R} 2765 \mathrm{C})$, which is predicted to be likely hypomorphic. ${ }^{15-17}$ Frameshift variant PKD1 c.11554delC is a recently reported variant with a clear pathogenic character. ${ }^{16} P K D 1$ analysis in genomic DNA from the father was unavailable.

Sequence analysis of the LRP5 gene yielded a unique LRP5 c.3468G > C; p.(Gln1156His) (rs724159825) in the proband which was absent in the DNA from both parents. The LRP5 variant p.(Gln1156His) was absent in dbSNP and all aforementioned online and in-house exome sequence datasets. This missense variant was predicted to be damaging and the amino acid is located between blades 5 and 6 of the fourth $\beta$-propeller domain. Glutamine may interact by hydrogen bonds with adjacent arginine, but shows hydrophobic features at this position. Histidine contains an imidazole functional group which stereometrically changes the position. The major effect of this missense variant is the change in electrostatic charge which may result in disturbed interactions at the surface of the protein domain.

\section{Functional studies}

Confocal imaging studies identified co-localization of LRP5 in the endoplasmic reticulum, but no differences were identified between LRP5 localization compared with the WT LRP5 construct for all four LRP5 variants (Figure 1).

Luciferase activity assays of variant LRP5 compared with WT LRP5 constructs were conducted to estimate Wnt signaling defects. Addition of ligand Wnt3a reduced Wnt signaling activity in LRP5 variants p.(Trp560Cys), p.(Arg1036Gln) and p.(Gln1156His) (Figure 2). Wnt signaling activation showed an increasing tendency for AXIN2 and LEF1 gene expression (Supplementary Figures). LRP5 variant 
a

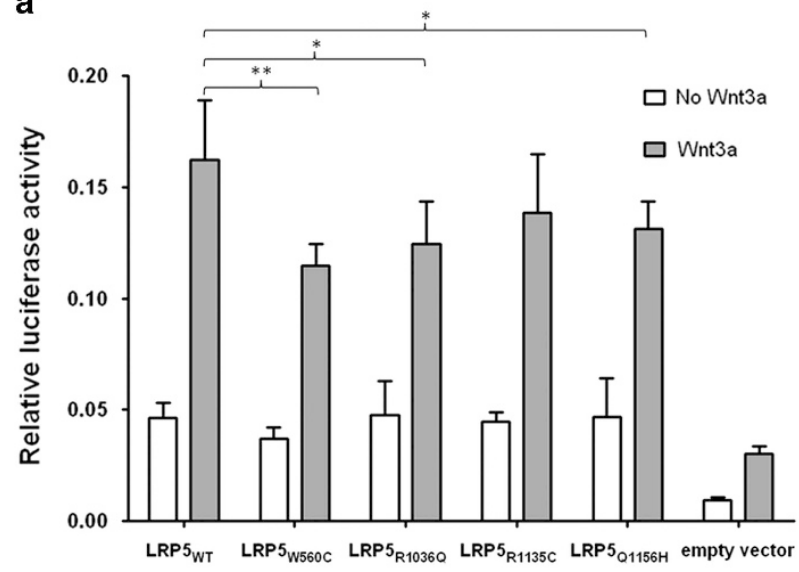

b
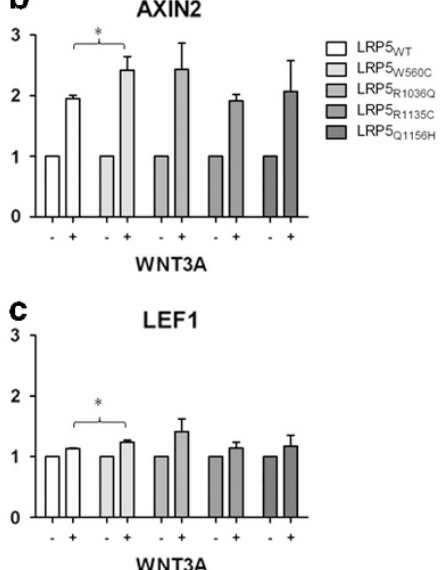

Figure 2 LRP5 variants present reduced activation of canonical Wnt signaling. (a) Canonical Wnt signaling activity was analyzed by firefly luciferase activity and normalized to renilla luciferase activity without (white bar) and with (grey bar) addition of $250 \mathrm{ng} / \mathrm{ml}$ Wnt3a. LRP5 constructs showed a significant increase in Wnt signaling activity compared with the empty vector. LRP5 variants p.(Trp560Cys), p.(Arg1036GIn) and p.(GIn1156His) presented a decreased Wnt3a-induced signal activity $\left({ }^{*} P<0.05 ; * * P<0.01\right)$. (b) All LRP5 variants indicated only concordantly increased AXIN2 expression levels by Wnt3a activation. (c) $L E F 1$ and others showed slightly increased gene expression. Significant increased AXIN1 and LEF1 expression by Wnt3a activation was present in $L R P 5$ variant p.(Trp560Cys) compared with $\angle R P 5$ WT $\left({ }^{*} P<0.05\right)$. The $y$-axis presents the relative gene expression level.

p.(Trp560Cys) compared with LRP5 WT presented significant increased AXIN1 and LEF1 expression by Wnt3a activation.

\section{DISCUSSION}

The data presented here suggest a role of LRP5 in ADPKD. We discovered two unique and two rare LRP5 variants in ADPKD patients regardless of family history or presence of $P K D$ mutations. The association of $L R P 5$ variants with hepatic cystogenesis was identified in an extended PCLD pedigree. ${ }^{13}$ The fact that polycystic livers are present in up to $94 \%$ of ADPKD patients ${ }^{2}$ prompted us to investigate the possible pathogenic role of LRP5 variants in ADPKD patients. There are a number of functional and animal studies that support the thinking that LRP5 variants may contribute to hepatic and renal cystogenesis.

LRP5 is a single transmembrane co-receptor involved in the canonical Wnt signaling pathway that orchestrates mammalian organogenesis. Northern blotting studies indicate that LRP5 is strongly expressed in both liver and kidneys. ${ }^{18}$ In addition, there is abundant LRP5 expression in convoluted tubules of human and mouse kidneys. ${ }^{19}$ Canonical Wnt signaling is established by binding of a Wnt ligand to a cognate Frizzled receptor in the presence of LRP5. The role of canonical Wnt signaling in ADPKD has been an area of research. ${ }^{20-25}$

Increased canonical Wnt signaling ${ }^{20-22}$ and diminished signal activation or stable $\beta$-catenin ${ }^{23-25}$ that lead to an opposite effect of planar cell polarity/noncanonical signaling have been reported in different ADPKD mouse models. These data suggest renal cystogenesis is initiated by imbalance between canonical and planar cell polarity/ non-canonical signaling. ${ }^{26}$ Indeed, polycystins modulate Wnt activity and disruption of either pathway may lead to polycystic kidney disease. $^{20,21}$ During the late stage of nephron maturation, polycystins are required for terminal differentiation of epithelial cells. ${ }^{27}$ This led to the model that put the canonical $/ \beta$-catenin pathway as one of the downstream effectors for ADPKD. ${ }^{28}$

A targeted knock-out LRP5 mouse develops hepatobiliary abnormalities without specified hepatic nor renal morphology, ${ }^{24}$ although it is known that LRP5 models have limited severity of Wnt disease phenotype compared with the almost identical LRP6 gene. ${ }^{29}$
The LRP6 knock-out mouse model is lethal, in view of severe developmental defects that affect skeleton, retina and ureter (E8.5). At E18.5, macroscopic small cystic kidneys are visible, suggesting a PKD phenotype. ${ }^{24}$ These findings support the notion that Wnt signaling is pivotal during early kidney development while polycystins appear to be critical during final stages of renal tubulogenesis. ${ }^{28}$

This paper provides evidence that the discovered variants affect LRP5 function. Luciferase activity assays of four LRP5 constructs decrease Wnt3a-induced signal activation across various cell lines (Figure 2). ${ }^{13}$ In addition, the expression of AXIN2, a Wnt signaling gene is enhanced in the presence of three LRP5 mutants (Supplementary Figure S5). This suggests that there is constitutive activation of the Wnt signaling as a result of these LRP5 variants.

Loss or gain of function of genes involved in Wnt signaling may lead to an imbalance between canonical and noncanonical signaling events. ${ }^{30}$ There is evidence that key players of Wnt signaling modulate the renal disease severity in ADPKD by antagonizing canonical Wnt signaling. ${ }^{5,6}$ Three single nucleotide polymorphisms in dickkopf Wnt signaling pathway inhibitor 3 (DKK3) downregulate canonical Wnt signaling similar to our data for LRP5. ${ }^{5}$ In addition, HNF-1 $\beta$ which orchestrates Wnt signaling may aggravate congenital renal cystogenesis in mice and human autosomal recessive polycystic kidney disease and ADPKD.,17 Failure of canonical Wnt signaling homeostasis will result in activated noncanonical signaling. It is likely that other genes or modifier genes in the Wnt signaling pathway are linked to hepatic and renal cystogenesis. ${ }^{20}$

Multiple $P K D$ variants may cause early and more severe renal disease in ADPKD. ${ }^{6}$ Although the pathogenic character of both PKD1 variants in family A remains unclear, we surmise that the resulting inframe deletion of an alanine amino acid neighboring another alanine does not significantly affect the protein domain structure. A PKD1 missense variant p.(Val1045Met) $(c .3133 G>A)$ in the fifth PKD domain has been predicted to be likely hypomorphic. ${ }^{31}$ At the similar sequence position, we detected PKD1 c.3133G $>C$ leading to a hydrophobic amino acid change. Leucine and methionine are different regarding presence of a methyl group in leucine and a sulfide molecule in methonine, which are both larger components compared with amino acid valine. 
The case is different for the PKD1 variants identified in proband 101 from family D. PKD1 c.8293C>T; p.(Arg2765Cys) is present in an unaffected parent, but also is probably hypomorphic and weakly conserved. ${ }^{15,32}$ The second PKD1 variant, c.11554delC; p.(Leu3852Trpfs ${ }^{\star 93}$ ), is detected by next-generation sequencing and probably causative for the ADPKD phenotype. ${ }^{16}$ This family had a negative history for ADPKD that suggests a de novo onset of the PKD1 gene variant which has been described before. ${ }^{12}$ In order to detect the additional mutational effect of a LRP5 variant to the PKD1 c.1154delC, investigation of the severity of clinical ADPKD phenotype and subsequent functional studies are required.

Previous studies provided evidence that the type of $P K D 1$ variant correlates strongly with renal survival. Carriers of a truncating PKD1 variant have an earlier age at onset of end-stage renal disease which supports the concept of a genotype-phenotype correlation in ADPKD. ${ }^{33}$

In conclusion, several in vitro and in vivo studies suggest involvement of aberrant Wnt signaling and unregulated $\beta$-catenin levels contribute to renal cystogenesis. ${ }^{23,24}$ Our findings are in line with the hypothesis that genes encoding $\mathrm{Wnt} / \beta$-catenin signaling partners may be candidate loci for cystic disorders. ${ }^{13,20}$ We postulate that LRP5 variants may render ADPKD patients more susceptible to the development of polycystic liver although further studies are needed to validate our findings.

\section{CONFLICT OF INTEREST}

WRC is supported by a grant of the Institute for Genetic and Metabolic Disease (IGMD) of the Radboud university medical center and by a grant from the Dutch foundation of digestive diseases WO1050 (MLDS). The authors declare no conflict of interest.

\section{ACKNOWLEDGEMENTS}

We thank our patients and their families for their participation and providing samples. We appreciate the input of the Genomic Disorders Group from the Department of Human Genetics, Radboud university medical center. We thank Mrs Irene Otte-Höller, Department of Pathology; Mr Ing. Huib Croes, Department of Cell Biology, Radboud university medical center for advise and expertise. Dr ADM Stork, internal medicine (St Anna Ziekenhuis Geldrop) and Dr A Bökenkamp, pediatric nephrologist (VUmc Amsterdam) for sharing clinical information. This work was funded by the Institute of Genetic and Metabolic Diseases (IGMD) of the Radboud university medical center and by a grant from the Dutch foundation of digestive diseases (MLDS; WO10-50). These study sponsors were not involved in study design or in collection, analysis, and interpretation of data.

1 Torres VE, Harris PC, Pirson Y: Autosomal dominant polycystic kidney disease. Lancet 2007; 369: 1287-1301.

2 Bae KT, Zhu F, Chapman AB et al: Magnetic resonance imaging evaluation of hepatic cysts in early autosomal-dominant polycystic kidney disease: the Consortium for Radiologic Imaging Studies of Polycystic Kidney Disease cohort. Clin J Am Soc Nephrol 2006; 1: 64-69.

3 Luciano RL, Dahl NK: Extra-renal manifestations of autosomal dominant polycystic kidney disease (ADPKD): considerations for routine screening and management. Nephrol Dial Transplant 2014; 29: 247-254.

4 Torra R, Darnell A, Estivill X et al: Interfamilial and intrafamilial variability of clinical expression in ADPKD. Contrib Nephrol 1995; 115: 97-101.

5 Liu M, Shi S, Senthilnathan S, Yu et al: Genetic variation of DKK3 may modify renal disease severity in ADPKD. J Am Soc Nephrol 2010; 21: 1510-1520.
6 Bergmann C, von Bothmer J, Ortiz Bruchle $\mathrm{N}$ et al: Mutations in multiple PKD genes may explain early and severe polycystic kidney disease. J Am Soc Nephrol 2011; 22: 2047-2056.

7 Rossetti S, Harris PC: Genotype-phenotype correlations in autosomal dominant and autosomal recessive polycystic kidney disease. J Am Soc Nephrol 2007; 18: 1374-1380.

8 The polycystic kidney disease 1 gene encodes a $14 \mathrm{~kb}$ transcript and lies within a duplicated region on chromosome 16. The European Polycystic Kidney Disease Consortium. Cell 1994; 77: 881-894.

9 Mochizuki T, Wu G, Hayashi T et al: PKD2, a gene for polycystic kidney disease that encodes an integral membrane protein. Science 1996; 272: 1339-1342.

10 Peters DJ, Sandkuijl LA: Genetic heterogeneity of polycystic kidney disease in Europe. Contrib Nephrol 1992; 97: 128-139.

11 Rossetti S, Consugar MB, Chapman AB et al: Comprehensive molecular diagnostics in autosomal dominant polycystic kidney disease. J Am Soc Nephrol 2007; 18: 2143-2160.

12 Reed B, McFann K, Kimberling WJ et al: Presence of de novo mutations in autosomal dominant polycystic kidney disease patients without family history. Am J Kidney Dis 2008; 52: 1042-1050.

13 Cnossen WR, Te Morsche RH, Hoischen A et al: Whole-exome sequencing reveals LRP5 mutations and canonical Wnt signaling associated with hepatic cystogenesis. Proc Natl Acad Sci USA 2014; 111: 5343-5348.

14 Pei Y, Obaji J, Dupuis A et al: Unified criteria for ultrasonographic diagnosis of ADPKD. J Am Soc Nephrol 2009; 20: 205-212.

15 Gout AM, Martin NC, Brown AF: PKDB: Polycystic Kidney Disease Mutation Databasea gene variant database for autosomal dominant polycystic kidney disease. Hum Mutat 2007: 28: 654-659.

16 Rossetti S, Hopp K, Sikkink RA et al: Identification of gene mutations in autosomal dominant polycystic kidney disease through targeted resequencing. J Am Soc Nephrol 2012; 23: 915-933.

17 Hiesberger T, Bai Y, Shao X et al: Mutation of hepatocyte nuclear factor-1beta inhibits Pkhd1 gene expression and produces renal cysts in mice. J Clin Invest 2004; 113: 814-825.

18 Hey PJ, Twells RC, Phillips MS et al: Cloning of a novel member of the low-density lipoprotein receptor family. Gene 1998; 216: 103-111.

19 Figueroa DJ, Hess JF, Ky B et al: Expression of the type I diabetes-associated gene LRP5 in macrophages, vitamin A system cells, and the Islets of Langerhans suggests multiple potential roles in diabetes. J Histochem Cytochem 2000; 48: 1357-1368.

20 Saadi-Kheddouci S, Berrebi D, Romagnolo B et al: Early development of polycystic kidney disease in transgenic mice expressing an activated mutant of the betacatenin gene. Oncogene 2001; 20: 5972-5981.

21 Qian CN, Knol J, Igarashi $\mathrm{P}$ et al: Cystic renal neoplasia following conditional inactivation of apc in mouse renal tubular epithelium. J Biol Chem 2005; 280: 3938-3945.

22 Happe H, Leonhard WN, van der Wal A et al: Toxic tubular injury in kidneys from Pkd1deletion mice accelerates cystogenesis accompanied by dysregulated planar cell polarity and canonical Wnt signaling pathways. Hum Mol Genet 2009; 18: 2532-2542.

23 Miller MM, Iglesias DM, Zhang Z et al: T-cell factor/beta-catenin activity is suppressed in two different models of autosomal dominant polycystic kidney disease. Kidney Int 2011; 80: 146-153.

24 Pinson KI, Brennan J, Monkley S et al: An LDL-receptor-related protein mediates Wnt signalling in mice. Nature 2000; 407: 535-538.

25 Marose TD, Merkel CE, McMahon AP et al: Beta-catenin is necessary to keep cells of ureteric bud/Wolffian duct epithelium in a precursor state. Dev Biol 2008; 314: $112-126$.

26 Lancaster MA, Gleeson JG: Cystic kidney disease: the role of Wnt signaling. Trends Mol Med 2010; 16: 349-360.

27 Lu W, Peissel B, Babakhanlou $\mathrm{H}$ et al: Perinatal lethality with kidney and pancreas defects in mice with a targetted Pkd1 mutation. Nat Genet 1997; 17: 179-181.

$28 \mathrm{Kim}$ E, Arnould T, Sellin LK et al: The polycystic kidney disease 1 gene product modulates Wnt signaling. J Biol Chem 1999; 274: 4947-4953.

29 Fujino T, Asaba H, Kang MJ et al: Low-density lipoprotein receptor-related protein 5 (LRP5) is essential for normal cholesterol metabolism and glucose-induced insulin secretion. Proc Natl Acad Sci USA 2003; 100: 229-234.

30 Simons M, Gloy J, Ganner A et al: Inversin, the gene product mutated in nephronophthisis type II, functions as a molecular switch between Wnt signaling pathways. Nat Genet 2005; 37: 537-543.

31 Vujic M, Heyer CM, Ars E et al: Incompletely penetrant PKD1 alleles mimic the renal manifestations of ARPKD. J Am Soc Nephrol 2010; 21: 1097-1102.

32 Rossetti S, Kubly VJ, Consugar MB et al: Incompletely penetrant PKD1 alleles suggest a role for gene dosage in cyst initiation in polycystic kidney disease. Kidney Int 2009; 75: 848-855.

33 Cornec-Le Gall E, Audrezet MP, Chen JM et al: Type of PKD1 mutation influences renal outcome in ADPKD. J Am Soc Nephrol 2013; 24: 1006-1013.

Supplementary Information accompanies this paper on European Journal of Human Genetics website (http://www.nature.com/ejhg) 